\title{
Advanced vectorial simulation of VCSELs with nano structures invited paper
}

\author{
Chung, II-Sug; Mørk, Jesper
}

Published in:

9th International Conference on Numerical Simulation of Optoelectronic Devices, 2009. NUSOD 2009

Link to article, DOI:

10.1109/NUSOD.2009.5297222

Publication date:

2009

Document Version

Publisher's PDF, also known as Version of record

Link back to DTU Orbit

Citation (APA):

Chung, I-S., \& Mørk, J. (2009). Advanced vectorial simulation of VCSELs with nano structures invited paper. In 9th International Conference on Numerical Simulation of Optoelectronic Devices, 2009. NUSOD 2009 (pp. 6566). IEEE. https://doi.org/10.1109/NUSOD.2009.5297222

\section{General rights}

Copyright and moral rights for the publications made accessible in the public portal are retained by the authors and/or other copyright owners and it is a condition of accessing publications that users recognise and abide by the legal requirements associated with these rights.

- Users may download and print one copy of any publication from the public portal for the purpose of private study or research.

- You may not further distribute the material or use it for any profit-making activity or commercial gain

- You may freely distribute the URL identifying the publication in the public portal 


\title{
Advanced vectorial simulation of VCSELs with nano structures
}

\author{
invited paper \\ Il-Sug Chung and Jesper Mørk \\ Department of Photonics Engineering, Technical University of Denmark, DK-2800 Kgs. Lyngby, \\ Denmark, ilch@fotonik.dtu.dk
}

\begin{abstract}
The single-mode properties and design issues of three vertical-cavity surface-emitting laser (VCSEL) structures incorporating nano structures are rigorously investigated. Nano structuring enables to deliver selective pumping or loss to the fundamental mode as well as stabilizing the output polarization state. Comparison of three vectorial simulation methods reveals that the modal expansion method is suitable for treating the nano structured VCSEL designs.
\end{abstract}

Index Terms - subwavelength grating, photonic crystal, HCG

\section{INTRODUCTION}

High single-mode output power of vertical-cavity surface-emitting lasers (VCSELs) can be of high interest in many applications. Various approaches have been investigated to increase the single-mode output power, e.g. [1]-[3]. Among them, employing nano structures looks promising since it can not only improve single-mode output power but also stabilize the polarization state. The optical simulations of the nano structured VCSELs should be vectorial in order to accurately treat scattering, diffraction, and polarization-related effect.

This presentation consists of two parts: In the former part, three vectorial simulation methods for VCSELs are compared in terms of computational speed, memory demand, and capability of quantitatively measuring various optical losses. In the latter part, the single mode properties of three nano structured VCSEL designs are investigated: a photonic crystal $(\mathrm{PhC})$ VCSEL, a long wavelength VCSEL with an intra-cavity air gap, and a high-index-contrast sub-wavelength grating (HCG) VCSEL.

\section{COMPARISON OF VECTORIAL METHODS}

Three vectorial methods compared in this section are the finite element method (FEM), the finite-difference time-domain method (FDTD), and a modal expansion method with coupled mode approach [1]. The FEM and FDTD use spatial grids to express a field distribution within a computational domain of interest while the modal expansion method uses a linear combination of basis functions, i.e., expansion coefficients of the basis functions. For VCSEL simulations, the modal expansion approach is much faster and requires less memory than the spatial grid approaches, as summarized in Table I. This is because the number of expansion coefficients involved is typically much smaller than TABLE I

COMPARISON OF VECTORIAL METHODS

\begin{tabular}{cccc}
\hline \hline & FEM [4] & FDTD & Modal expansion \\
\hline Simulation time* $^{*} 10-30 \mathrm{hrs}$ & $10 \mathrm{hrs}-2 \mathrm{wks}$ & A few sec $-3 \mathrm{hrs}$ \\
Memory & $20-50 \mathrm{~GB}$ & $30-130 \mathrm{~GB}$ & $0.1-3 \mathrm{~GB}$ \\
\hline \hline
\end{tabular}

* Simulation time taken to find a mode.

that of spatial grids. As shown in Fig. 1(a), the field distribution

(a)
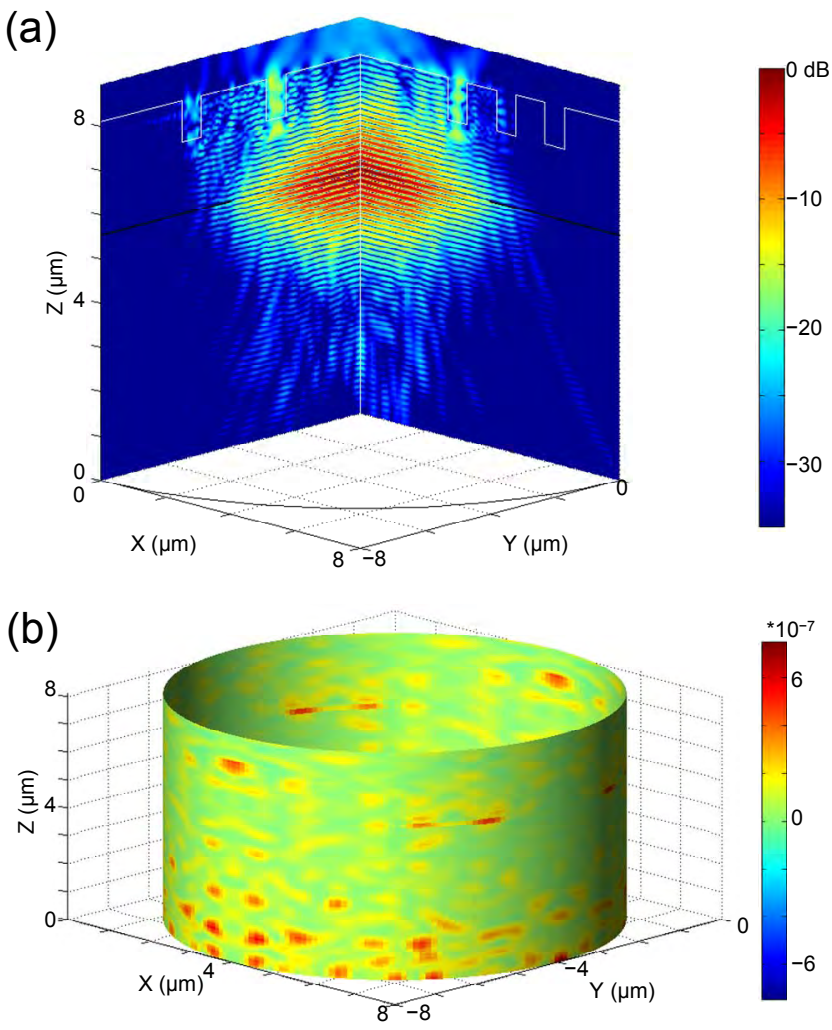

Fig. 1. (a) A normalized intensity profile $|E|^{2}$ and (b) a normalized radially-outward Poynting vector profile of the fundamental mode of a VCSEL structure with photonic crystal air holes in the top DBR.

within a VCSEL structure can be obtained precisely by using the modal expansion method.

The modal expansion method can also quantitatively estimate the scattering loss due to nano structures and the absorption loss in the active region; this evaluation is not feasible using the spatial grid approaches. It is because the 


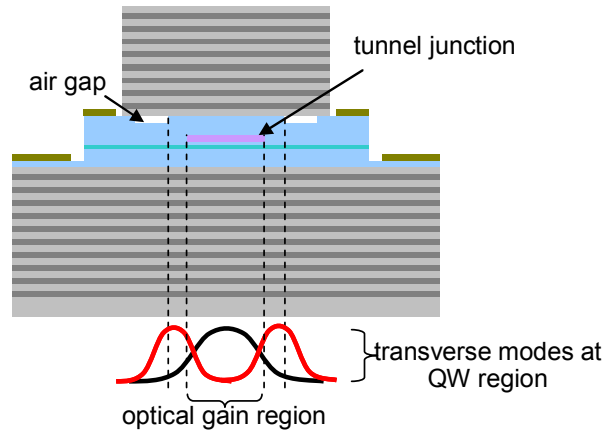

Fig. 2. Schematic diagram of the long wavelength VCSEL with a tunnel junction and an intra-cavity air gap.

spatial grid approach cannot decompose the upward and downward propagating modes, which is necessary for this evaluation. Fig. 1(b) shows a radially-outward Poynting vector profile that is used for the measurement of scattering loss. This capability of measuring the scattering and absorption losses is essential to understand the single mode mechanisms, as discussed in the next section.

In conclusion, the modal expansion approach is adequate for the simulation of nano structured VCESLs, due to its numerical efficiency and capability of estimating various losses.

\section{RESUlTS AND DISCUSSIONS}

Using the modal expansion method, the single mode properties of three VCSEL structures are rigorously investigated.

\section{A. Photonic crystal VCSEL}

The single-mode operation of the PhC VCSEL relies on the high scattering loss of higher order modes. As shown in Fig. 1(a), strong scattering occurs near the air holes, due to the large index difference. The total scattering loss of the first order mode is 2.7 times larger than that of the fundamental mode; the fundamental mode becomes dominant.

The scattering loss of the fundamental mode can be reduced while keeping the single mode operation, by replacing the semiconductor Bragg mirror with a dielectric Bragg mirror [1].

\section{B. Long wavelength VCSEL with an intra-cavity air gap}

The single-mode operation results from the selective pumping of the fundamental mode. As schematically shown in Fig. 2, the gain region and the transverse mode profiles are independently determined by the tunnel junction and by the air gap, respectively. This makes it possible that most of optical gain is delivered to the fundamental mode. Higher order modes experience high absorption loss.

The key issues to be solved are the non-uniformity of polarization state of output across a wafer and its instability as a function of injection current. They are closely related to a lithographic misalignment of the intra-cavity air gap and a thermal lensing, respectively. Simulations show that employing a surface or intra-cavity subwavelength grating can effectively remove both problems.

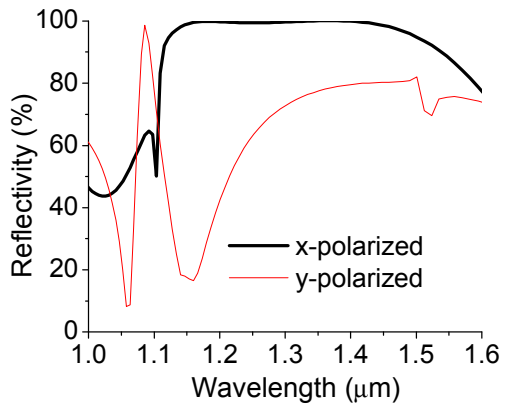

Fig. 3. Reflectivity of the investigated HCG vs. wavelength. The HCG is highly reflective to the x-polarized electric field whose is polarized perpendicular to the grating groove directions.

\section{Subwavelength grating-mirror VCSEL}

The reflectivity of a HCG is sensitive to the polarization of the incident field, e.g., as shown in Fig. 3. This property leads to strong polarization stability.

The moderate single-mode strength of a HCG VCSEL can be greatly enhanced by optimizing the HCG region size. When the size of the HCG region becomes similar as that of gain region, the single-mode strength becomes $2-3$ times stronger than a typical single-mode VCSEL structure [7].

\section{CONCLUSION}

Among several vectorial methods, the modal expansion approach is desirable for simulations of nano structured VCSEL designs, due to its high numerical efficiency and capability of estimating various losses. Nano structuring can greatly enhance the strength of single mode operation by delivering selective loss or pumping to the fundamental mode as well as stabilizing the polarization state, promising high single-mode output power.

\section{ACKNOWLEDGMENT}

This work has been supported by EU IST-FP6 through the MOSEL project and by FTP grant of the Danish Research Council (Sagsnr: 274-08-0361). Authors thank to V. Iakovlev and his colleagues for providing a test device structure.

\section{REFERENCES}

[1] D. Zhou, "High-power single-mode antiresonant reflecting optical waveguide-type vertical-cavity surface-emitting lasers," IEEE J. of Quant. Electron., vol. 38, pp. 1599-1605, 2002.

[2] Å. Haglund, et al., "Single fundamental-mode output power exceeding 6 $\mathrm{mW}$ from VCSELs with a shallow surface relief," IEEE Photonic. Technol. Lett., vol. 16, pp. 368-370, 2004.

[3] A. Furukawa, et al., "High-power single-mode vertical-cavity surface-emitting lasers with triangular holey structure," Appl. Phys. Lett., vol. 85, pp. 5161-5163, 2004.

[4] P. Nyakas, "Full-vectorial three-dimensional finite element optical simulation of vertical-cavity surface-emitting lasers," J. Lightwave Technol. 25, 2427-2434 (2007).

[5] G. P. Bava, P. Debernardi, and L. Fratta, "Three-dimensional model for vectorial fields in vertical-cavity surface-emitting lasers," Phys. Rev. A vol. 63, p. 023816, 2001.

[6] I.-S. Chung, et al., "Vectorial analysis of dielectric photonic crystal VCSEL," International Conference on Transparent Optical Networks, Mo.D1.2, 2009.

[7] I.-S. Chung, et al., "Subwavelength grating-mirror VCSEL with a thin oxide gap," IEEE Photonic. Technol. Lett., vol. 20, pp. 105-107, 2008. 